\title{
Violencia y emergencia obstétrica desde la perspectiva de las parteras tradicionales en comunidades rurales
}

\author{
Violence and obstetric emergencies from the perspective of \\ traditional midwives in rural communities
}
Violência e emergência obstétrica a partir da perspectiva de parteiras tradicionais em comunidades rurais

\section{Carlos Alberto Aranda-Téllez* , Hugo Yam Chalé** y Hans Oudhof***}

\begin{abstract}
RESUMEN
Comprender los significados construidos en torno a la violencia obstétrica permite identificar factores de riesgo, permitiendo generar estrategias de prevención efectivas. En 2016 el 33,3\% de las mujeres mexicanas experimentó algún tipo de violencia obstétrica en su último parto, provocando efectos en la salud materna y repercutiendo en mayores complicaciones obstétricas. El presente estudio tuvo como objetivo identificar formas de violencia obstétrica causales de emergencias obstétricas, a partir de los relatos de las parteras tradicionales de comunidades rurales del estado de Veracruz, México. El tema se abordó desde el construccionismo social. Se condujeron 8 grupos focales con 52 parteras tradicionales, registrados en dos programas institucionales de salud. Se identificaron agresiones directas del personal médico y procedimientos médicos significados como violentos. Algunas formas de violencia identificadas en los relatos fueron agresiones
\end{abstract}

Palabras clave: embarazo, medicina

tradicional, partera, servicio de salud, violencia contra la mujer.

\footnotetext{
* Mexicano. Doctorante en Investigación Psicológica. Universidad Iberoamericana. Ciudad de México, México. miquiztli@icloud.com / ORCID: https://orcid.org/00000003-3474-860X

** Mexicano. PhD. Profesor de Tiempo Completo en la Universidad Iberoamericana. Ciudad de México, México. hugo.yam@ibero.mx / ORCID: https://orcid.org/0000-00026879-3815

*** Mexicano. PhD. Profesor de tiempo completo en Universidad Autónoma del Estado de México. Toluca, México. hansovb@hotmail.com / ORCID: https://orcid.org/00000003-1265-3052
} 
física-emocionales, tacto vaginal y cesárea innecesaria. Dichas formas de violencia favorecen la resistencia de las usuarias a la atención institucional y conducen a situaciones de emergencia obstétrica. Significar una institución de salud agresora y como un factor de riesgo para la madre podría reforzar conductas de rechazo a los servicios hospitalarios y provocar situaciones de emergencia obstétrica que comprometan la vida de la madre y el neonato.

\begin{abstract}
Understanding the meanings constructed around obstetric violence allows us to identify risk factors, making it possible to devise effective prevention strategies. In 2016, 33.3\% of Mexican women experienced some form of obstetric violence during their most recent delivery, having an impact on maternal health and leading to further obstetric complications. The aim of this study was to identify forms of obstetric violence that cause obstetric emergencies, based on the accounts of traditional midwives in rural communities in the state of Veracruz, Mexico. The topic was approached from a social constructionist perspective. Eight focus groups were formed with 52 traditional midwives, registered in two institutional health programs. They identified direct aggression by medical personnel as well as medical procedures that were considered to be violent. Some forms of violence identified in the accounts were physical-emotional abuse, vaginal touching and unnecessary caesarean section. These forms of violence foster a reluctance of patients to seek institutional care and lead to obstetric emergencies. If health institutions come to be seen as an abusive environment and a risk factor for the mother, this could reinforce the rejection of hospital services and trigger emergency obstetric situations that could compromise the life of the mother and the newborn.
\end{abstract}

\section{RESUMO}

A compreensão dos significados construídos em torno da violência obstétrica nos permite identificar fatores de risco e gerar estratégias de prevenção eficazes. Em 2016, 33,3\% das mulheres mexicanas sofreu alguma forma de violência obstétrica em seu último parto, causando efeitos na saúde materna e resultando em maiores complicações obstétricas. O objetivo do presente estudo foi identificar formas de violência obstétrica como causa de emergências obstétricas, com base nos relatos de parteiras tradicionais em comunidades rurais no estado de Veracruz, México.
Keywords: pregnancy, traditional medicine, midwife, health service, violence against women.

Palavras-chave: gravidez, medicina tradicional, parteira, serviço de saúde, violência contra a mulher. 
O tema foi abordado a partir da perspectiva do construcionismo social. Oito grupos focais foram conduzidos com 52 parteiras tradicionais, registrados em dois programas institucionais de saúde. Foram identificadas agressões diretas da equipe médica e procedimentos médicos considerados violentos. Algumas formas de violência identificadas nos relatos foram agressões físicas e emocionais, toque vaginal e cesárea desnecessária. Estas formas de violência favorecem a resistência das usuárias ao atendimento institucional e levam a situações de emergência obstétrica. $\mathrm{O}$ fato de uma instituição de saúde ser vista como um agressor e como um fator de risco para a mãe poderia reforçar comportamentos de rejeição aos serviços hospitalares e provocar situações de emergência obstétrica que comprometam a vida da mãe e do recém-nascido. 


\section{Introducción}

Dos situaciones desafortunadas que encontramos en torno al parto son la violencia obstétrica y la emergencia obstétrica. Por violencia obstétrica podemos entender todo acto de violencia (intencional o no intencional) sufrido por las mujeres durante la atención del parto en los centros de salud institucional (United Nations, 2019), y emergencia obstétrica se refiere a las condiciones de salud que ponen en peligro la vida de la mujer y/o del neonato, que requiere atención médica y/o quirúrgica de manera inmediata (Secretaría de Salud, 2009). En la presente investigación se estudia, desde la perspectiva de las parteras tradicionales, los significados construidos en torno a la violencia obstétrica ejercida en las mujeres gestantes que atienden, y que provocan conductas de resistencia a recibir los servicios de salud institucional y en algunos casos provocar situaciones de emergencia obstétrica.

En 2016, el 33,3\% de las mujeres mexicanas experimentó algún tipo de violencia obstétrica en su último parto (Castro y Frías, 2019), lo cual tiene efectos en la salud materna y neonatal, por lo que las complicaciones maternas son más altas en las mujeres que han experimentado violencia durante su embarazo (Romero-Gutiérrez et al., 2011). Se ha comprobado que las actitudes negativas hacia el embarazo y las dificultades de atención percibidas por las mujeres mexicanas son determinantes en complicaciones durante el parto (Quelopana et al., 2009), al igual que un estado emocional de miedo puede tener un impacto negativo en el progreso de su trabajo de parto (Bak, 2003).

En este sentido, la normalización de la violencia en ocasiones hace poco visible ciertas formas de maltrato y dificulta dimensionarla con claridad (Ramírez, 2018). Al respecto, se ha estudiado que en ocasiones las mujeres que son víctimas de violencia obstétrica a menudo son silenciadas o tienen miedo de hablar por temor a los tabúes, el estigma o por la creencia de que la violencia que han sufrido corresponde solo a un acto aislado (United Nations, 2019); es decir, formas naturalizadas de abuso que pueden no ser interpretadas como tales por los prestadores de servicios de salud y, a veces, por las propias mujeres (Castro y Erviti, 2015).

Esta normalización de la violencia obstétrica forma parte de un sistema de violencia simbólica, la cual puede tomar formas diversas 
y refinadas, pero que tiene siempre como efecto la desvalorización, el empobrecimiento cultural y la sumisión de sus portadoras (Bourdieu et al., 1998); y esta solo se puede instaurar con la complicidad de quienes las padecen a través de su afección o de su admiración por el explotador (Bourdieu, 2007). Cabe mencionar que la violencia simbólica ejercida por la institución de salud sobre las mujeres embarazadas hace referencia a un plano simbólico-subjetivo; sin embargo, ello no significa la minimización o descarte de la violencia física ejercida de manera directa, indirecta o, en este caso, por medio de una intervención quirúrgica (Bourdieu y Jordà, 2000).

En este mismo sentido, muchas de las mujeres que padecen violencia simbólica piensan que esta es causa de su propia responsabilidad, ya que son culpabilizadas de los hechos y estas introyectan esta culpa como suya, por medio de un discurso de poder ejercido por la institución médica a través de su personal de salud y, ante ello, pareciera que contra la violencia simbólica no hay defensa (Bourdieu et al., 1998).

Por otro lado, se ha encontrado que en comunidades marginadas las mujeres prefieren dar a luz en su casa en lugar del hospital (Amanuel et al., 2019); un estudio realizado en comunidades indígenas de México reveló que de 62 mujeres entrevistadas, 33 dieron a luz en su casa sin asistencia calificada y 5 dieron a luz completamente solas en sus hogares, y en estos eventos 5 neonatos murieron durante el parto o el período perinatal (Gamlin y Holmes, 2018). Esta preferencia de algunas mujeres en zonas rurales o indígenas por parir en su casa, se puede atribuir a diversos factores, que podrían ser culturales, de accesibilidad a los servicios de salud, desconocimiento o a una resistencia a aceptar los servicios de salud institucional, debido a una experiencia de maltrato o violencia. Sin embargo, también se ha evidenciado que el parto es una intensa experiencia psicológica y transformadora, que genera empoderamiento, y mejora su creencia en su capacidad de generar un nacimiento sin necesidad de alguna intervención médica (Olza et al., 2018).

En comunidades rurales e indígenas es común consultar médicos tradicionales por razones de salud, pero también para obtener consejos acerca de sus problemas personales (Berenzon-Gorn et al., 2006). Las parteras tradicionales son personas pertenecientes a comunidades indígenas o rurales que atienden el embarazo, parto, puerperio y 
cuidado del recién nacido; su trabajo consiste en acompañar, reconfortar y atender a la mujer embarazada aplicando métodos tradicionales (Álvarez y Miranda Orrego, 2014). Al estar insertas en el mismo contexto cultural y geográfico de las mujeres que atienden, conocen la cosmovisión del lugar y su relación está definida horizontalmente (Valdez-Santiago et al., 2004); es decir, pertenecen a una cultura médica popular compleja protagonizada principalmente por las mujeres de la comunidad (Osorio Carranza, 2001).

Anteriormente, se ha estudiado la familiaridad de las parteras tradicionales con la violencia que viven las mujeres embarazadas de manera cotidiana (Valdez-Santiago et al., 2004), y se ha visto que se han convertido en un soporte emocional, de confianza para estas mujeres y de compañía durante su proceso de gestación en lo físico, en lo emocional en las repercusiones implicadas en el embarazo y dinámica familiar; así como en lo subjetivo relacionado con los usos y costumbres de la localidad; a la vez de que representan un actor de referencia para la construcción de significados en torno al parto, la violencia y la institución.

Posteriormente a la inauguración del hospital regional del municipio de Coscomatepec Veracruz, la institución de salud estableció una “interrelación" con las parteras tradicionales en la región, denominándolas como un personal no profesional autorizado para la prestación de los servicios de atención médica (Secretaría de Salud, 2017). De esta forma, las parteras tradicionales son ubicadas como mediadoras entre la embarazada y la institución de salud, lo que las convierte en informantes clave que concentran los relatos de las mujeres embarazadas y de la institución de salud, lo cual redunda en una práctica muy conveniente para la institución de salud, mientras esta no cuente con la posibilidad de extender su cobertura en las zonas más marginadas (Menéndez, 2018).

Esta mediación entre la mujer embarazada y la institución de salud adquirió especial relevancia en embarazos identificados como de alto riesgo (preclamsia, placenta previa, estrechez uterina, etc.), que de acuerdo con la institución deben de ser notificados y canalizados al hospital para su inmediata intervención. Sin embargo, aunque mayoritariamente la norma se cumple, las parteras refieren que mucho antes de la llegada del hospital estos casos eran atendidos por las par- 
teras tradicionales, con quienes las mujeres embarazadas se sentían más seguras de ser atendidas.

Estos cambios establecidos por la llegada de la institución de salud en la dinámica social han significado la resignificación de la partera tradicional, su representación ante la comunidad y la forma en la que son percibidas por las usuarias, generando nuevos significados respecto de la salud materna, el embarazo y el parto. En este sentido, la partera tradicional produce influencia de manera activa en los significados construidos por parte de las mujeres gestantes, las que pueden manifestarse en conductas y actitudes de la mujer embarazada hacia la institución de salud, al igual que su familia y la comunidad.

El principal obstáculo que enfrenta la investigación en torno a la violencia obstétrica es la conceptualización que se hace de la misma: desde el campo médico se perciba como un problema de "calidad de la atención" (Castro y Erviti, 2014), manifestándose en actos negligentes, imprudentes, omisos, discriminatorios e irrespetuosos (Olza et al., 2018), y no como actos plenos de violación a los derechos humanos (United Nations, 2019). En este sentido, esta investigación partió desde los significados construidos por las parteras tradicionales en torno a la violencia obstétrica y lo que estas identifican como maltrato institucional, al mismo tiempo que integran este aspecto a su esquema de percepciones y genera conductas.

De esta forma, esta investigación tuvo como objetivo identificar las formas de violencia obstétrica en mujeres embarazadas y su vínculo con situaciones de emergencia obstétrica, a partir de los relatos elaborados por las parteras tradicionales. Contar con esta información puede generar conocimientos de la situación que viven en sus comunidades. Con ello, se espera aportar a la generación de políticas públicas y estrategias de acción para prevenir riesgos en materia de salud obstétrica, en un marco de respeto por la cultura, costumbres y prácticas comunitarias, generando estrategias de intervención pertinentes, pero resguardando la integridad de las mujeres gestantes, condiciones de género, derechos humanos y derecho a una vida reproductiva sin violencia (United Nations, 2019).

La realización de este estudio siguió un método cualitativo de tipo fenomenológico, que pretende estudiar las realidades como se presen- 
tan, permitiendo que se manifiesten por sí mismas, sin ajustarlas a una estructura externa determinada (Martínez-Miguelez, 2014), de modo de analizar casos concretos en su particularidad temporal y local y - a partir de las expresiones y actividades de las personas en sus contextos locales (Flick, 2012)—, así lograr el entendimiento de una realidad mediante un proceso interpretativo (Reese et al., 2012). El trabajo investigativo se realizó desde el construccionismo social, enfoque que postula que las personas construimos significados de nosotros mismos, nuestras experiencias y nuestro entorno a través de la interacción con los otros y la construcción de una realidad colectiva (Gergen y Gergen, 2011); es decir, la forma en la que percibimos el mundo y actuamos en él (Watzlawick y Machain, 1998). En este sentido, el origen de la construcción de significados radica en la interacción humana, en un acto de negociación de conceptos socialmente aceptados, ya que sin acuerdos negociados en cuanto a los medios y propósitos de la vida organizativa, el sistema resultaría desequilibrado (Gergen, 2018).

\section{Metodología}

El estudio se realizó de octubre 2018 a junio 2019, tiempo en el que se contactó a las parteras tradicionales de la región a partir del censo del Instituto Mexicano del Seguro Social-Bienestar (IMSS-B) y con apoyo del personal (promotores comunitarios) encargados de este programa. Este organismo del gobierno federal tiene como finalidad proporcionar servicios de primer y segundo nivel de atención a personas que viven en zonas rurales o urbanas marginadas (Gobierno de México, 2020). De igual forma, se trabajó a partir del censo de la Secretaría de Salud del Estado de Veracruz (SSVer) organismo del gobierno estatal que ofrece servicios de salud a los pobladores de este estado (Veracruz Gobierno del Estado, 2020), y con el apoyo del personal encargado del programa de partería tradicional.

Las parteras tradicionales participantes provenían de 22 poblaciones de 6 municipios del estado de Veracruz, México y se encuentran registradas en su totalidad en alguno de los sistemas de salud mencionados. Para los fines de esta investigación se registraron las narraciones de las parteras tradicionales, indistintamente del organismo de salud en el que se encontraran registradas (IMSS-Bienestar o SSVer), ya que en una misma comunidad existen parteras tradicionales registradas en 
diferentes instituciones; de esta forma, se priorizó el factor geográficosocial por sobre el registro institucional. En este sentido, ambas instituciones aceptaron facilitar la investigación, así como respetar el código ético propio del estudio.

La selección de parteras fue por conveniencia. Como criterios de inclusión se consideró que se encontraran registradas en alguna de las instituciones de salud mencionadas, que tuvieran por lo menos un año de ejercer como parteras y de interrelación con la institución de salud, que no contaran con estudios obstétricos formales, que fueran reconocidas por la institución y que su edad o estado de salud no fuera un impedimento para asistir y participar en los grupos focales. Por su parte, como criterios de exclusión se consideró la no aceptación voluntaria y/o el abandono de la participación. Se tuvo contacto con 90 parteras, de las cuales 52 cumplieron con los criterios de inclusión; la media de edad fue 56 años, con 33 años de ejercer la partería (máx. 63-mín. 1) y una media de 502 partos atendidos por cada una; las participantes se encontraron en promedio a $16 \mathrm{~km}$ de distancia del centro de salud más cercano (máx. 52 km - mín. 1 km); en este sentido, la distancia se consideró un factor relevante para interpretar las condiciones de acceso de las mujeres embarazadas a los sistemas de salud y la canalización de los casos de emergencia. Los detalles de esta muestra son expuestos con mayor precisión en la Tabla 1. 


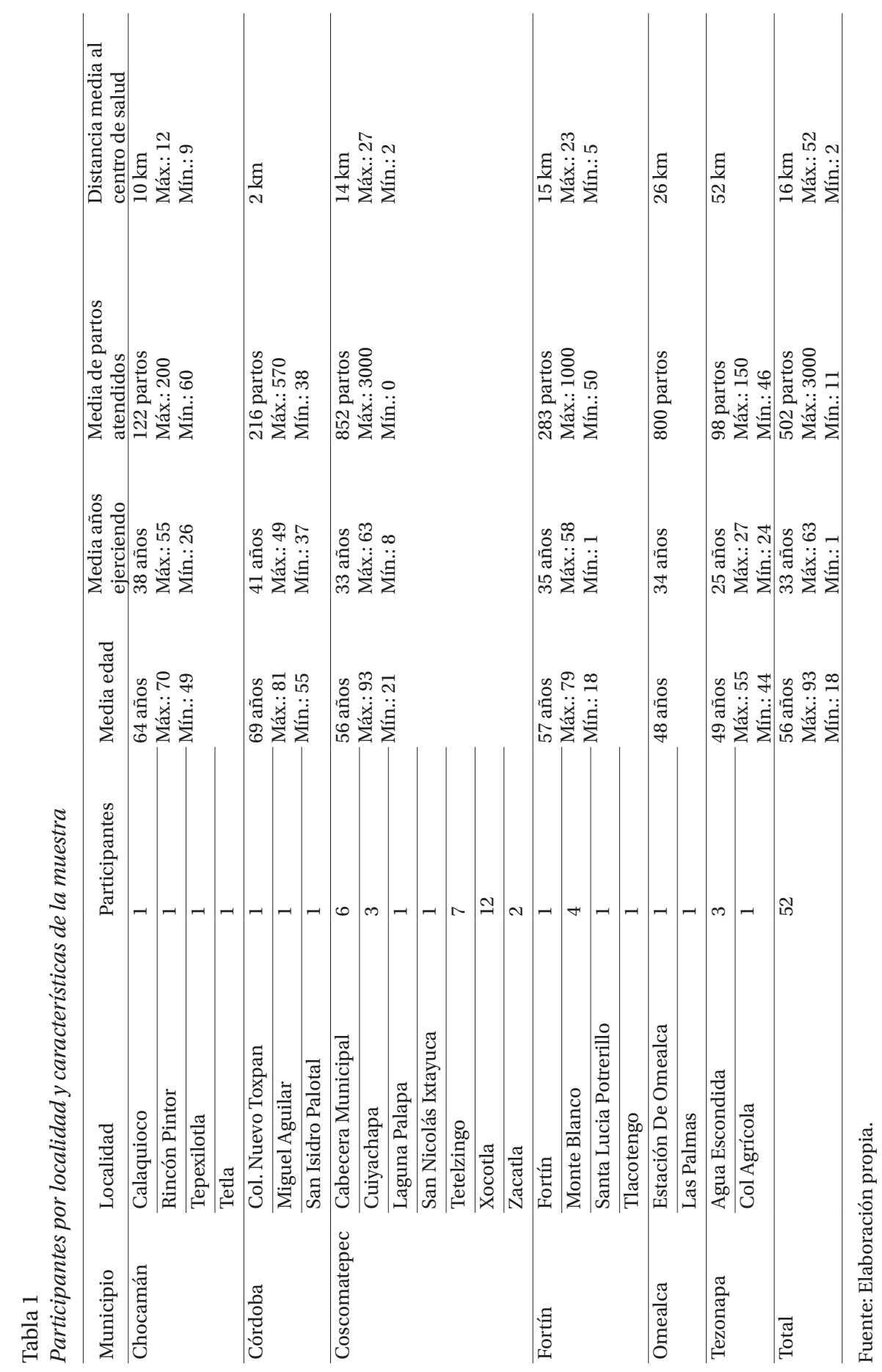


La información se recabó a través de la observación participante (Guasch, 2002) y grupos focales (Stake, 2015), orientados por una guía de entrevista semiestructurada previamente diseñada. El número de entrevistas realizadas se determinó siguiendo el criterio de saturación teórica (Soneira, 2006); se siguieron como ejes temáticos los significados construidos acerca de:

a) La relación con la institución, la cual permitió identificar cómo es la interrelación y de qué forma se da la coordinación en materia de salud obstétrica.

b) El ejercicio de la partería tradicional, para conocer cuál es su aportación desde lo social y cultural, así como sus referentes respecto de la salud obstétrica.

c) La relación con la mujer embarazada y su familia, que permitió conocer cómo se dan los lazos de confianza y desde qué perspectiva se entiende el maltrato institucional.

Las sesiones de grupos focales se celebraron en privacidad (Kamberelis y Dimitriadis, 2015), en un espacio seguro en donde las participantes se sintieran cómodas, importantes y legitimadas. Por estrategia se eligió un aula del centro de salud más próximo a la comunidad de las participantes, ya que se trataba de un lugar familiar para ellas, conocían las vías de acceso y era el punto en común más próximo a su lugar de residencia. Las sesiones fueron audiograbadas previa explicación y autorización en la carta de consentimiento informado (Flick, 2015); luego fueron transcritas en su totalidad para su posterior análisis. Por último, con el fin de proteger la identidad de las participantes, sus nombres fueron sustituidos por un código de identificación para el control de los datos. El análisis de información se realizó siguiendo una codificación inductiva (Martínez-Miguelez, 2014), la cual consistió en partir de una codificación de sistema abierto y, a medida que fueron progresando, las categorías se hicieron más definitivas y completas, hasta acabar con un sistema cerrado de codificación (Ruiz Olabuénaga, 2012), lo cual fue posible gracias al apoyo del software ATLAS.ti 7.0, especializado en análisis cualitativo.

La consistencia interna se procuró mediante la triangulación constante de la información con la teoría y participantes diferentes (Flick, 2014). Como estrategia de la gestión de la calidad de la investigación 
se realizó una devolución de los resultados a siete participantes (Goetz y LeCompte, 2010), con las cuales se expuso y explicó cada uno de los conceptos resultados. De esta forma, y por medio de una escala Likert, evaluaron si los resultados correspondían a su realidad y si se encontraban de acuerdo con la interpretación de sus narraciones; en su gran mayoría refirieron estar de acuerdo y totalmente de acuerdo con los hallazgo obtenidos.

En todo el estudio se aplicaron los principios éticos de investigación (Moscoso y Díaz, 2017). El estudio se apegó a lo estipulado en la Declaración de Helsinki, así como al ordenamiento de la Ley General en materia de Investigación para la salud de México. Con marco en esos instrumentos el estudio se clasificó como de riesgo mínimo, toda vez que no se realizó ninguna intervención o modificación intencionada en las variables fisiológicas, psicológicas y sociales de los individuos que participaron en la investigación. Una vez explicados los objetivos y procedimientos del estudio, así como atendidas las dudas de las participantes y obligaciones del investigador, se solicitó por escrito el consentimiento informado a todas las participantes y se garantizó la confidencialidad sobre la identidad de las informantes.

\section{Desarrollo}

La violencia contra la mujer embarazada referida por las parteras tradicionales se clasificó en dos grupos: agresiones derivadas de la institución, actos producto de una agresión directa del personal institucional; y la vivencia de procedimientos médicos, los cuales podrían partir de una recomendación estrictamente médica o de un acto negligente, aunque la usuaria lo viva como una agresión indistintamente. Cada uno de estos grupos se encuentra integrados por los tipos diferentes que se describen a continuación.

\section{Agresiones derivadas de la institución}

Se atribuyen directamente como consecuencia de un acto u omisión directa de la institución. Las categorías que integran este grupo son: agresión física-verbal; rechazo institucional y valoración inadecuada; insuficiencia de recursos humanos y condiciones institucionales. 


\section{Agresión física-verbal}

Las agresiones físicas o verbales son identificadas por las parteras como "maltratos". Las agresiones verbales giran en torno a descalificativos o afirmaciones con referentes sexuales que, normalmente, las hace sentir trasgredidas. Las agresiones físicas son identificadas como golpes experimentados en su cuerpo y/o actos violentos en su trabajo de parto.

ese doctor cuando atendía un parto las nalgueaba muy feo, porque las mujeres estaban gritando, y les decía "cuando están con el marido ni siquiera chistan y ahorita ya estas gritando", y les daba unas nalgadas. (PT047).

En este mismo sentido, cuando estas agresiones provienen de una mujer se significan como un tipo de violencia mayor, al existir con el personal institucional femenino una expectativa de empatía del sentido de ser mujer y ser madre.

hay unas doctoras que son mujeres, saben cómo se siente tener un hijo, aún así dicen "órale pújale, vente, no grites; a la hora de la hora te estaba gustando todo eso" [refiriendo la relación sexual]. Es una mujer que se lo está diciendo a otra mujer, eso no se dice, son cosas que no deben ser. (PT022).

\section{Rechazo institucional y valoración inadecuada}

Se da cuando la mujer embarazada acude al centro de salud para exploración o evidente trabajo de parto y se le rechaza o se realiza una valoración inadecuada de su estado. Este tipo de situaciones son significadas por las parteras tradicionales como una falta de cooperación institucional, una carencia de sensibilidad o empatía y una discriminación, al considerar que la valoración de la partera carece de importancia y sustento; y cuando la partera considera que es un caso de urgencia obstétrica, en este rechazo ve un innecesario incremento de riesgo de una complicación y la situación se exacerba. Al mismo tiempo, la partera refiere sentirse impotente de proteger a la mujer embarazada, por ello cuando la embarazada es acompañada por la partera tradicional —en algunas ocasiones- resulta en un conflicto con el personal médico.

"la pacientita que cité a tales horas, que se regrese, porque no la puedo atender", entonces agarré yo y dije: doctor, si usted no puede 
atenderlo [el parto], entonces yo puedo atenderlo afuera, porque ya lo sentí, que ya viene, entonces yo atiendo a mi nuera afuera, yo también puedo y también sé. (PTx68).

[quisiera que] cuando lleguemos al hospital con nuestras embarazadas nos deben recibir, porque a veces no la reciben porque no hay camas. También uno se siente mal que le digan que no, o ver que los niños nacen ahí en el baño o afuera en el pasillo. (PT067).

\section{Insuficiencia de recursos humanos y condiciones institucionales}

Aunado a lo anterior, las participantes identificaron una insuficiencia de personal capacitado para situaciones de emergencia, lo que en algunas ocasiones deriva en un rechazo institucional.

me dijeron "si te quieres esperar, espérate, porque no hay doctor, y aquí firma, porque si te llega a pasar algo a ti o al bebé, nosotros no nos hacemos responsables". (PT087).

Con relación a las condiciones institucionales las parteras hicieron referencia a la insuficiencia de higiene en las que se encuentran algunas instalaciones; mismas que interpretan como una situación incongruente al recibir críticas de parte del personal médico referente a las condiciones en las que las parteras tradicionales atienden en sus casas.

\section{Vivencia de procedimientos médicos}

Se refiere a la vivencia de la mujer embarazada durante las intervenciones médicas, las cuales son vividas de forma corporal con repercusiones emocionales. Estos procedimientos médicos pueden ser una recomendación justificada y estrictamente médica, o bien, parte de un acto negligente del personal institucional, sin embargo, la usuaria lo vive como una agresión directa indistintamente del origen. Los tipos que integran este grupo son: cesárea; tacto vaginal; OBT o salpingoclasia; episiotomía; y maniobra de Kristeller.

\section{Cesárea}

Referida como "la rajaron", "le dieron cuchillo", "la cesaria”. Las parteras identificaron que la cesárea es una práctica común y necesaria en casos de feto transverso, placenta previa o cuello uterino estrecho. Sin embargo, indican que en muchas de las ocasiones la cesárea responde 
más bien a razones de tiempo o de practicidad institucional, ya que de esa forma no es necesario esperar el parto y la intervención quirúrgica se realiza en menos de 30 minutos. A este respecto las parteras significan un estado de indefensión ante la institución de la mujer embarazada y de ella misma, así como también un peligro de trasgresión a sus creencias y su forma de hacer el parto, además del latente riesgo de alguna otra forma de violencia obstétrica.

El acomodamiento de feto es una práctica común entre las parteras. Refieren que esta práctica en algunos casos evita que se practique una cesárea debido un mal posicionamiento fetal. Por ello, señalan que algunas embarazadas deciden acudir con la partera a escondidas de la institución, para así realizarse acomodamiento fetal y evitar la intervención quirúrgica.

\section{Tacto vaginal}

Referida como "dedeando". Se refiere a la práctica manual para identificar el grado de dilatación, el posicionamiento del feto y la etapa del trabajo de parto, identificándola como algo común y que se realiza sin el cuidado y la privacidad necesaria. Incluso, cuando hay algún grupo de estudiantes haciendo residencia, las mujeres son revisadas por ellos provocando inflamación y en ocasiones complicaciones en el parto. Debido a esta situación prefieren esperarse con la partera hasta tener una dilatación adecuada para llegar al hospital ya en pleno trabajo de parto, evitando el constante chequeo, lo cual conlleva el riesgo de una atención demorada.

[Las mujeres dicen que las revisan] cada 20 minutos, cada media hora, "¡ora! ya parezco gallina ponedora” y con la partera no, porque está con calma, con paciencia. (PT049).

\section{OBT (oclusión tubaria bilateral) o salpingoclasia}

Referida como "la cesárea chiquita", "la otra cesaría para no tener hijos", "para quitarle la maternidad". Se trata del método definitivo de anticoncepción consistente en la oclusión de las trompas de Falopio (United Nations, 2019). Las parteras tradicionales refirieron el miedo de las embarazadas a que se les practique este método sin su consentimiento. A este respecto, se identificó que es común que relacionen o confundan la cesárea con la salpingoclasia, la cual es significada 
frecuentemente como una "pérdida de la maternidad". En este sentido, cuando esta operación es realizada sin consentimiento la significan como una transgresión mayor. De igual forma, señalan que en algunas ocasiones son engañadas para firmar la carta de consentimiento para la realización de la intervención quirúrgica; y en este sentido, la anticoncepción inducida y sin consentimiento puede ser revisada y analizada como una práctica sistemática contra las mujeres racializadas, en una violación de los derechos reproductivos de las mujeres, pero en ocasiones más sutil y normalizada, en un procedimiento sistemático y argumentado como necesario para la mujer (Castro y Erviti, 2015).

a veces haciendo la cesárea, le quitan la maternidad a la mamá, porque le hacen la otra cesárea para no criar, a veces con permiso, y a veces sin permiso. (PT067).

\section{Episiotomía}

Referido como "cortesito" y "la rajadita". Consiste en un corte profundo en el perineo de la mujer que llega hasta el músculo del suelo pélvico; se realiza para ayudar quirúrgicamente a la mujer que va a tener un parto vaginal (United Nations, 2019). Las participantes indicaron comprender que, en algunas ocasiones, esta práctica puede ser recomendada, sin embargo indicaron que esto les genera confusión principalmente en tres sentidos: en primer lugar, la consideran innecesaria cuando se trata de un parto en buenas condiciones; en segundo lugar se conflictúa con su concepto de pujar, ya que para ellas este acto es un proceso en el que "una mujer se convierte en madre", es común en sus comunidades la frase "yo pujé a mis hijos"; y en tercer lugar, la latente posibilidad de una mala práctica de esta intervención y que esta tenga repercusiones en su salud física y sexual (United Nations, 2019). Las participantes atribuyeron a esta situación como parte de la causa de la resistencia de las usuarias a la intervención hospitalaria, es decir, al asistir a los servicios de salud institucional la mujer embarazada se enfrenta desde el principio a tres inminentes amenazas: cesárea, salpingoclasia o episiotomía; independientemente de que sea una recomendación médica necesaria o no, las participantes las refirieron como situaciones de inminente intervención y una posible mala práctica en alguna de ellas. 
[Pregunta la muchacha] “¿Por qué nos van hacer el cortesito? Si nosotras podemos pujar". Veo que eso es, eso lo que teme la embarazada, ahí van las pobres niñas a tener su bebé, les hacen el corte, que es muy doloroso, que les duele mucho. (PT022).

\section{Maniobra de Kristeller}

Referido como "le apachurran la panza", consiste en la presión manual del fondo uterino durante la segunda fase del alumbramiento, con el fin de acelerar el parto (United Nations, 2019). Aunque las parteras indicaron que no es una práctica frecuente, sí se encuentra muy presente en su imaginario colectivo, probablemente por el impacto emocional que significa en las parteras tradicionales que les tocó vivenciar esta práctica, además de considerarla como una causa directa de muerte neonatal. En este sentido, de acuerdo con los relatos de las participantes, estas se sienten responsables de estos actos, ya que fueron ellas quienes canalizaron a la mujer embarazada a los centros de salud, indicando que en ocasiones se sienten culpabilizadas por las usuarias, de manera que lo viven como un acto de traición, que fractura el lazo de confianza existente entre la partera tradicional y la mujer embarazada.

de que les aplastan la panza, les matan los niños, se ahogan, se ahogan los niños, hay veces que sacan dos o tres muertos y como para ellos no hay delito. (PT019).

decía, sentía un dolor, sentía que me iba morir, dice y usted tiene la culpa por no atenderme, le digo pero es que eres de alto riesgo, ¿qué hago contigo? (PTx45).

\section{Resistencia a la atención institucional y la emergencia obstétrica}

En atención de los relatos de las parteras tradicionales se observa que estas modalidades de violencia obstétrica son los principales motivos de resistencia a la atención institucional por parte de las usuarias, misma que puede derivar en una situación de emergencia obstétrica o en otras condiciones que pueden comprometer la vida de la embrazada y el neonato.

No querían salir, vino la ambulancia y la patrulla y los sacaron a fuerza, a la señora la subieron a fuerza, que ya quería tener a su bebé ahí, a fuerza la subieron, y al esposo igual a fuerza, se los llevaron a los dos, pero a fuerza (PT031). 
Las participantes refirieron que algunas mujeres embarazadas consideran un mayor riesgo recurrir a los servicios institucionales que buscar otras alternativas para parir. Indicaron que esta situación coloca a las parteras en un dilema: por un lado, ser empáticas con la mujer gestante, comprender sus miedos y su mala experiencia en la institución de salud; y por otro, identificar y reconocer un embarazo o parto de alto riesgo que precisa intervención quirúrgica inmediata. En este contexto, la partera tradicional refiere sentirse imposibilitada por la institución de salud de hacer frente a tal emergencia obstétrica, por lo que decide acompañar a la mujer embarazada a la institución de salud para que reciba inmediata atención. Acompanándose y desconociendo ambas si se enfrentarán a un rechazo institucional, personal capacitado suficiente, una baja capacidad de reacción ante la emergencia, o una discriminación hacia la partera tradicional. Esto coloca a usuarias y parteras en un terreno circundante de amenazas constantes, por ello en ocasiones refieren que la partera tradicional es el único lugar seguro para la embarazada y su hijo.

La verdad hay muchas mujeres que por ser maltratadas no quieren ir al hospital, por eso tienen a sus hijos solas en sus casas, aunque puedan morirse. Pero es necesario que algunas de ellas vayan al hospital, porque las que son de alto riesgo, por ejemplo las que tienen su hijo transverso, las que son anémicas, ¿qué vamos hacer nosotras con ellas? (PT064).

Las parteras señalaron haber conocido varios casos en los que los procesos administrativos resultaron ser un difícil andamiaje que genera situaciones adversas en la atención, que junto con la resistencia a la atención institucional de parte de la mujer embarazada, favorecen las condiciones de emergencia obstétrica que comprometen la vida de la mujer embarazada y del neonato.

hay ocasiones que en el hospital no las quieren atender pronto, porque luego hay unas señoras que tienen a su niño afuera, en el piso lo tienen, que ya van con los dolores y tiran la criatura abajo. (PT031). 


\section{Discusión y conclusiones}

El rechazo institucional y la valoración errónea se presentó como la forma más frecuente de violencia hacia la mujer. Esto se ha estudiado en otros contextos mexicanos e identificado como el fenómeno de multi-rechazo hospitalario (Collado y Sánchez, 2016), que se refiere a las complicaciones que presentan las mujeres por consecuencia de una atención demorada. En este sentido, el rechazo institucional se manifestó como uno de los principales factores que provocan que la mujer embarazada evite los servicios de salud institucional, provocando la aparición de situaciones de emergencia obstétrica de acuerdo con los relatos de las participantes, debido a que desde sus conceptos la atención hospitalaria no es adecuada, es transgresora y falta de empatía con la mujer embarazada y el embarazo, además de estar fuera del contexto sociocultural de su localidad. En este caso, el ejercicio pleno de la violencia simbólica desde la superioridad institucional sobre la vulnerabilidad de la usuaria se verifica a partir de un discurso médico de su personal sanitario (Bourdieu, 2007); una lógica del mecanismo social de represión en la que los médicos buscan obligar a las mujeres a que "se controlen", se callen, o dejen de quejarse, "recordándoles" que en otro momento seguramente experimentaron placer sexual y que "por lo tanto", ahora deben vivir "con las consecuencias" (Castro y Erviti, 2015).

De esta forma, también se observó que las actitudes negativas hacia el embarazo y las barreras percibidas de atención médica entre mujeres mexicanas son un factor de complicaciones obstétricas (Quelopana et al., 2009). De igual forma, la insuficiencia de personal capacitado en las instituciones de salud se mantuvo muy presente en las parteras tradicionales, lo que coincide como una problemática generalizada a nivel mundial, por el daño que causa en las mujeres embarazadas (United Nations, 2019). En México existen estudios que confirman esa carencia de personal y habilidades para la atención obstétrica (Méndez-Hernández et al., 2003), lo que puede dar sentido al temor de las mujeres embarazadas de acercarse a los servicios de salud institucional y que el parto ocurra sin una asistencia adecuada (PeronGiaxa y Silva-Marques Ferreira, 2011). Así el rechazo institucional, la valoración errónea, la carencia de recursos y el maltrato a las usuarias podrían conformar una presente, pero poco visible forma de violencia institucional (Bellamy y Castro, 2019). 
Estas condiciones permiten concluir que los significados construidos por las parteras tradicionales no distan mucho de la realidad objetiva a nivel global. En este sentido, al significar que una institución de salud es agresora, necesaria pero insensible a los significados locales, con baja reacción ante la urgencia obstétrica y con una carencia de recursos humanos; podría reforzar las conductas de rechazo hacia los servicios de salud y su personal por parte de las usuarias.

Cabe recalcar que las parteras tradicionales normalmente son líderes de opinión ante su comunidad y con la mujer que les consulta y deposita su confianza en ellas, por lo que su opinión tiene peso en la construcción de significados de la mujer embarazada respecto de la institución. Esta opinión puede ser reforzada por una mala experiencia de la usuaria y consolidar así la idea de que la institución de salud representa un factor de riesgo para la embarazada, más que ser un recurso de ayuda; provocando así una falta de identificación oportuna de embarazos de alto riesgo, así como la prevención de emergencias obstétricas que conduzcan a situaciones que comprometan la vida de la madre y del neonato.

La práctica de la cesárea es significada como uno de los principales temores y resistencias a la atención institucional para las mujeres embarazadas. El programa IMSS-Prospera reportó haber atendido en el 2018 más del 29\% de partos por cesárea (Instituto Mexicano del Seguro Social, 2018), cuando la OMS ha reportado que una tasa normal de cesáreas debe oscilar entre el 10\% y 15\% (Organización Mundial de la Salud, OMS, 2015a), y aunado a esto existe un mayor riesgo de mortalidad materna en una cesárea en comparación con el parto vaginal (Fahmy et al., 2018).

Así mismo, las parteras tradicionales refirieron que la práctica del tacto vaginal ya es significada como una violación a su intimidad por sí misma y que es maximizada la experiencia si no se realiza en condiciones adecuadas de discreción y cuidado, pues termina por empeorar si esta es mal practicada y deriva en alguna complicación obstétrica. Las participantes refirieron que el tacto vaginal en los centros de salud se realiza con frecuencia cada 20 minutos, y según la OMS el tacto vaginal se debe realizar en intervalos de cuatro horas para valoración de rutina e identificación de la prolongación del trabajo de parto activo (OMS, 2015b). 
Respecto de la violencia verbal hacia una mujer embarazada, esta se identificó como una práctica generalizada. Así, en varias partes del mundo se han descrito prácticas profundamente humillantes, agresiones verbales y observaciones sexistas durante la atención del parto, que tienen lugar tras las puertas cerradas de los centros de salud (United Nations, 2019). Esto también fue evidenciado en este estudio y significado por las parteras como factores de rechazo a la atención institucional. De igual forma hay que considerar el factor de normalización de la violencia, que en ocasiones podría estar invisibilizando otras formas violencia (Ramírez, 2018).

En cuanto a la esterilización, esta se encuentra presente en los relatos de las participantes, referida como "perder la maternidad". Sin embargo, cuando esta práctica se realiza sin el consentimiento de la mujer y su familia, se transforma en "me quitaron la maternidad", y esto adquiere mayor relevancia, ya que en su contexto la fertilidad es significada con frecuencia como una de las principales virtudes femeninas, su capacidad de "dar vida" y conformar una familia; por lo que esta práctica - que probablemente sea parte de una estrategia poblacional o de salud-, puede generar repercusiones emocionales y fracturas en las estructuras básicas de sentido de vida, de acuerdo con el esquema de significados de las parteras tradicionales, teniendo implicaciones emocionales personales, sociales y hacia el interior de la familia.

De igual forma, respecto de la episiotomía, realizada sin el consentimiento de las mujeres y sin anestesia, fue relevada por las parteras tradicionales, puesto que atienden partos sin recurrir a esta práctica, la significan como innecesaria y de fuertes implicaciones para la salud de la mujer embarazada. Al igual que la maniobra Kristeller, no es recomendada por la OMS, pero continúa siendo un procedimiento generalizado a nivel mundial (United Nations, 2019). En este caso, fue mencionada como una práctica que ya no es común en estas instituciones de salud, sin embargo sigue presente en los relatos de las parteras tradicionales, en los cuales también hay que considerar la normalización de la violencia (Ramírez, 2018) que bien podría infravalorar la frecuencia de estas prácticas.

Además de diversos factores sociales y culturales, vivir una experiencia de maltrato incrementa en las mujeres embarazadas la resistencia a una atención institucional (Colombara et al., 2016). Las 
vivencias de estas mujeres en torno al parto y al nacimiento afectan directamente sus percepciones y creencias respecto de dichos eventos (Peron-Giaxa y Silva-Marques Ferreira, 2011). Estas vivencias retroalimentan y consolidan los significados instalados en el imaginario social, favoreciendo al rechazo de la atención institucional, repercutiendo en el incremento de partos de alto riesgo y/o en partos extrahospitalarios. De ahí la importancia de fomentar condiciones que generen confianza en las mujeres embarazadas (Borges et al., 2018) y que los profesionales de la salud consideren los efectos potenciadores de la experiencia psicológica del parto fisiológico (Olza et al., 2018).

Los significados construidos en torno a los servicios de salud institucional tienen influencia en las actitudes de las mujeres embarazadas, su familia y la comunidad de la que provienen. Por lo que se recomienda capacitar y empoderar a las parteras como agentes de salud y como parte de una estrategia de prevención, reconociendo la parte sociocultural que representan para su comunidad. De esta forma, dentro de un marco de respeto y libertad para ejercer su labor, estas podrán fomentar el trabajo colaborativo con el personal institucional (Pabon Varela et al., 2018), sin que esto implique una trasgresión a su esquema de creencias y costumbres locales.

Al reconocer a la partera tradicional como un líder de opinión para su comunidad, ella podría generar mejores actitudes y conductas de las usuarias hacia los servicios de salud, lo que resultaría en una mayor y mejor prevención de riesgos obstétricos, y en el incremento de una visión más clara para todas las partes involucradas de cuándo una intervención quirúrgica es médicamente necesaria, o cuándo se transforma en una negligencia médica, un acto de violencia obstétrica o una plena violación a los derechos humanos de las usuarias.

En este sentido, el hecho de que las mujeres gestantes se resistan a la intervención institucional no responde específicamente a un acto de necedad, sino que es una conducta que debería ser entendida más a fondo, comprender otras implicaciones más profundas asociadas a esta resistencia, que llega al grado de poner en riesgo su vida y la de su hijo. Culpabilizar a las embarazadas de una "simple necedad de no ir al hospital", culpabilizar a la partera por no cumplir su "obligación" de enviar a la mujer en trabajo de parto al hospital, y amenazar con represalias legales por la muerte materna o neonatal como consecuencia de 
esta resistencia pone en evidencia la relación asimétrica de poder que ejerce la institución de salud sobre la partera tradicional (Castro y Erviti, 2015) y revela una incapacidad institucional para hacer frente a la salud obstétrica, al buscar como estrategia la repartición de culpas por medio del ejercicio de la violencia simbólica, más que asumir responsabilidad por medio de políticas de salud más comunitarias que institucionales, que hagan frente a las muertes maternas y neonatales, y a la violencia obstétrica (cesáreas y episiotomías innecesarias, tratos inadecuados y carencias en sus tiempos de reacción ante la emergencia).

Ello, porque desmontarse del discurso de superioridad institucional y en su lugar hacer alianzas con la población y los actores estratégicos como las parteras y los médicos tradicionales lograría generar estrategias horizontales que se traduzcan en mejores acciones en materia de salud obstétrica regional.

Por último, el presente estudio se limitó a comunidades rurales de una región del estado de Veracruz, y aunque los resultados coinciden con datos nacionales e internacionales, no implica que los significados construidos en torno a la violencia obstétrica puedan ser generalizados a otras zonas geográficas, condiciones de acceso a la salud u otras culturas, como el caso de comunidades indígenas; ni tampoco que las condiciones de emergencia obstétrica sean las mismas o que respondan a las mismas causales. Por ello, se abre la posibilidad para futuras investigaciones en otras regiones y variables, con la finalidad de conocer las realidades locales e identificar patrones en común que permitan responder a la problemática de índole general, pero respetando también la particularidad de cada región, sus condiciones y su cultura.

\section{Referencias}

Álvarez, D. y Miranda Orrego, M. I. (2014). Oficios que cuidan la vida: Partería y curación. Iconos. Revista de Ciencias Sociales, 18(50), 149-160. https://doi.org/10.17141/iconos.50.2014.1434

Amanuel, H., Palazuelos, D., Reyes, A., Montaño, M., Flores, H., y Molina, R. L. (2019). "Morir en camino": Community narratives about childbirth care in rural Chiapas. Global Public Health, 14(3), 396-406. https://doi.org/10.1080/17441692.2 018.1512143 
Bak, C. (2003). The role of fear in the U.S. Birthing Process. Midwifery Today, 67(1), 7-24. https://midwiferytoday.com/mt-articles/ role-fear-u-s-birthing-process/

Bellamy, C. y Castro, R. (2019). Formas de violencia institucional en la sala de espera de urgencias en un hospital público de México. Revista Ciencias de la Salud, 17(1), 120-137. http://dx.doi. org/10.12804/revistas.urosario.edu.co/revsalud/a.7621

Berenzon-Gorn, S., Ito-Sugiyama, E., y Vargas-Guadarrama, L. A. (2006). Enfermedades y padeceres por los que se recurre a terapeutas tradicionales de la Ciudad de México. Salud Pública de México, 48(1), 45-56. https://doi.org/10.1590/ s0036-36342006000100008

Borges, L., Sixto Pérez, A., y Sánchez Machado, R. (2018). Influencia del desempeño de los profesionales de enfermería en la atención humanizada al parto. Revista Cubana de Enfermería, 34(2), 1-16. http://revenfermeria.sld.cu/index.php/enf/article/view/1426

Bourdieu, P. (2007). Razones prácticas: sobre la teoría de la acción. Anagrama.

Bourdieu, P. y Jordà, J. (2000). La dominación masculina. Editorial Anagrama.

Bourdieu, P., Passeron, J. C., Melendres, J., y Subirats, M. (1998). La reproducción: elementos para una teoría del sistema de enseñanza. Distribuciones Fontamara.

Castro, R. y Erviti, J. (2014). 25 años de investigación sobre violencia obstétrica en México. Revista CONAMED, 19(1), 37-42. https://www.medigraphic.com/cgi-bin/new/resumen. cgi?IDARTICULO=57515

Castro, R. y Erviti, J. (2015). Sociología de la práctica médica autoritaria: Violencia obstétrica, anticoncepción inducida y derechos reproductivos ( $1^{\text {a }}$ ed.). Universidad Nacional Autónoma de México, Centro Regional de Investigaciones Multidisciplinarias. http://biblioteca.clacso.edu.ar/Mexico/crimunam/20170511032438/pdf_1464.pdf

Castro, R. y Frías, S. M. (2019). Obstetric violence in Mexico: Results from a 2016 National household survey 1. Violence Against Women, 1(1), 1-18. https://doi.org/10.1177/1077801219836732

Collado, S. P. y Sánchez, Á. (2016). ¿Referencia y contrarreferencia o multi-rechazo hospitalario? Un abordaje cualitativo. Revista 
CONAMED, 21(1), 32-41. https://www.medigraphic.com/ cgi-bin/new/resumen.cgi?IDARTICULO=57686

Colombara, D. V., Hernández, B., Schaefer, A., Zyznieuski, N., Bryant, M. F., Desai, S. S., ... Mokdad, A. H. (2016). Institutional delivery and satisfaction among indigenous and poor women in Guatemala, Mexico, and Panama. PLOS ONE, 11(4), 1-17. https://doi.org/10.1371/journal.pone.0154388

Fahmy, W. M., Crispim, C. A., y Cliffe, S. (2018). Association between maternal death and cesarean section in Latin America: A systematic literature review. Midwifery, 59(1), 88-93. https:// doi.org/10.1016/j.midw.2018.01.009

Flick, U. (2012). Introducción a la investigación cualitativa. Fundación Paideia Galiza; Ediciones Morata.

Flick, U. (2014). La gestión de la calidad en investigación cualitativa. Ediciones Morata.

Flick, U. (2015). El diseño de investigación cualitativa. Ediciones Morata.

Gamlin, J. y Holmes, S. (2018). Preventable perinatal deaths in indigenous Wixárika communities: An ethnographic study of pregnancy, childbirth and structural violence. BMC Pregnancy and Childbirth, 18(243), 1-10. https://doi.org/10.1186/ s12884-018-1870-6

Gergen, K. J. (2018). El yo saturado: dilemas de identidad en el mundo contemporáneo. Editorial Paidós.

Gergen, K. J. y Gergen, M. M. (2011). Reflexiones sobre la construcción social. Editorial Paidós.

Gobierno de México. (2020). Instituto Mexicano del Seguro Social-Bienestar (IMSS-BIENESTAR) [Página gubernamental]. http:// www.imss.gob.mx/imss-bienestar

Goetz, J. P. y LeCompte, M. D. (2010). Etnografía y diseño cualitativo en investigación educativa. Ediciones Morata.

Guasch, Ó. (2002). Observación participante. Centro de Investigaciones Sociológicas. http://www.cis.es/cis/opencm/ES/3_publicaciones/colecciones/ver.jsp?id=9788474762402

Instituto Mexicano del Seguro Social, I. (2018). Informe al Ejecutivo Federal y al Congreso de la Unión Sobre la Situación Financiera y los Riesgos del Instituto Mexicano del Seguro Social 20172018. Instituto Mexicano del Seguro Social. http://www. 
imss.gob.mx/sites/all/statics/pdf/informes/20172018/21InformeCompleto.pdf

Kamberelis, G. y Dimitriadis, G. (2015). Grupos focales. En N. K. Denzin y Y. S. Lincoln (Eds.), Manual de investigación cualitativa $I V$ (pp. 494-532). Gedisa Editorial.

Martínez-Miguelez, M. (2014). Ciencia y arte en la metodología cualitativa. Editorial Trillas.

Méndez-Hernández, P., Valdez-Santiago, R., Viniegra-Velázquez, L., Rivera-Rivera, L., y Salmerón-Castro, J. (2003). Violencia contra la mujer: Conocimiento y actitud del personal médico del Instituto Mexicano del Seguro Social, Morelos, México. Salud Pública de México, 45(6), 472-482. https://doi. org/10.1590/s0036-36342003000600007

Menéndez, E. L. (2018). Poder, estratificación social y salud. Publicacions Universitat Rovira i Virgili. https://doi. org/10.17345/9788484246879

Moscoso, L. F. y Díaz, L. P. (2017). Aspectos éticos en la investigación cualitativa con niños. Revista Latinoamericana de Bioética, 18(34), 51-67. https://doi.org/10.18359/rlbi.2955

Olza, I., Leahy-Warren, P., Benyamini, Y., Kazmierczak, M., Karlsdottir, S. I., Spyridou, A., ... Nieuwenhuijze, M. J. (2018). Women's psychological experiences of physiological childbirth: A meta-synthesis. BMJ Open, 8(10), 1-11. https://doi.org/10.1136/ bmjopen-2017-020347

Organización Mundial de la Salud, OMS. (2015a). Declaración de la OMS sobre tasas de cesárea WHO/RHR/15.02. Organización Mundial de la Salud. https://apps.who.int/iris/ bitstream/handle/10665/161444/WHO_RHR_15.02_spa. pdf? sequence $=1$

Organización Mundial de la Salud, OMS. (2015b). Recomendaciones de la OMS para la conducción del trabajo de parto (1ra ed.). Ediciones OMS. https://apps.who.int/iris/bitstream/handle/10665/179906/9789243507361_spa.pdf?sequence=1

Osorio Carranza, R. M. (2001). Entender y atender la enfermedad: Los saberes maternos frente a los padecimientos infantiles. Instituto Nacional Indigenista; Instituto Nacional de Antropología e Historia; Centro de Investigaciones y Estudios Superiores en Antropología Social. 
Pabon Varela, Y., Cortina Navarro, C., Brito Ferreira, K. D., y Freyle Brito, Y. L. (2018). La partería tradicional en tres regiones del Magdalena-Colombia. Revista Cubana de Enfermería, 34(2), 1-14. http://revenfermeria.sld.cu/index.php/enf/article/ view/1657

Peron-Giaxa, T. E. y Silva-Marques Ferreira, M. de L. (2011). Miedo e inseguridad de la gestante durante el trabajo de parto como motivos para la demanda de internación precoz. Revista Investigación y Educación en Enfermería, 29(3), 363-369. https://www.redalyc.org/articulo.oa?id=105222406004

Quelopana, A. M., Champion, J. D., y Salazar, B. C. (2009). Factors predicting the initiation of prenatal care in Mexican women. Midwifery, 25(3), 277-285. https://doi.org/10.1016/j. midw.2007.04.008

Ramírez, A. R. (2018). El parto en los modelos público institucional y partería tradicional: Motivaciones de las mujeres del istmo oaxaqueño. En G. Freyermuth Enciso (Ed.), Los caminos para parir en México en el siglo XXI: experiencias de investigación, vinculación, formación y comunicación (pp. 48-55). Centro de Investigaciones y Estudios Superiores en Antropología Social; Asesoría, Capacitación y Asistencia en Salud, A.C.

Reese, L., Kroesen, K., y Gallimore, R. (2012). Cualitativos y cuantitativos, no cualitativos vs. Cuantitativos. En R. Mejía Arauz y S. A. Sandoval (Eds.), Tras las vetas de la investigación cualitativa: Perspectivas y acercamientos desde la práctica (pp. 39-75). ITESO. https://publicaciones.iteso.mx/libro/traslas-vetas-de-la-investigacion-cualitativa_94845/

Romero-Gutiérrez, G., Cruz-Arvizu, V. H., Regalado-Cedillo, C. A., y Ponce-Ponce de León, A. L. (2011). Prevalence of violence against pregnant women and associated maternal and neonatal complications in Leon, Mexico. Midwifery, 27(5), 750753. https://doi.org/10.1016/j.midw.2010.06.015

Ruiz Olabuénaga, J. I. (2012). Metodología de la investigación cualitativa. Publicaciones de la Universidad de Deusto.

Secretaría de Salud. (2009). Convenio General de Colaboración que celebran la Secretaría de Salud, el Instituto Mexicano del Seguro Social y el Instituto de Seguridad y Servicios Sociales de los Trabajadores del Estado para la Atención de las Emergen- 
cias Obstétricas (Núm. CV034 09; p. 18). SSA/IMSS/ISSSTE. http://cnegsr.salud.gob.mx/contenidos/descargas/SMP/ ConvenioEO.pdf

Secretaría de Salud. (2017). Guía para la autorización de las parteras tradicionales como personal de salud. SALUD, 1-11.

Soneira, A. J. (2006). La teoría fundamentada en los datos (Grounded Theory) de Glaser y Strauss. En I. Vasilachis de Gialdino (Ed.), Estrategias de investigación cualitativa (pp. 153-174). Gedisa Editorial.

Stake, R. (2015). Estudios de casos cualitativos. En N. K. Denzin y Y. S. Lincoln (Eds.), Manual de investigación cualitativa III (pp. 154-197). Gedisa Editorial.

United Nations. (2019). A human rights-based approach to mistreatment and violence against women in reproductive health services with a focus on childbirth and obstetric violence: Note / by the Secretary-General (Seventy-fourth session Núm. 71/170; p. 23). United Nations General Assembly. https://digitallibrary.un.org/record/3823698

Valdez-Santiago, R., Arenas-Monreal, L., y Hernández-Tezoquipa, I. (2004). Experiencia de las parteras en la identificación de mujeres maltratadas durante el embarazo. Salud Pública de México, 46(1), 56-63. https://doi.org/10.1590/s003636342004000100008

Veracruz Gobierno del Estado. (1 de marzo 2020). Secretaría de Salud de Veracruz (SESVER) [Página gubernamental]. https:// www.ssaver.gob.mx/

Watzlawick, P. y Machain, N. M. de. (1998). La realidad inventada: ¿Cómo sabemos lo que creemos saber? Gedisa Editorial. 